\title{
Chronic kidney disease in rural areas of Sri Lanka: an evolving catastrophe needing an urgent response
}

\author{
S Jayasinghe \\ Ceylon Medical Journal 2014; 59: 94-96
}

\begin{abstract}
A high prevalence of patients with chronic kidney disease (CKD) was noted from the North Central Province of Sri Lanka, at least before 1999 [1]. Sadly, the academic and health community appear to have disregarded this observation until much later [2]. Despite numerous subsequent publications, studies and reports, the epidemic continues its path of death and destruction, leaving behind devastated families, orphans, and destroyed communities in its wake. This process has the capacity to cripple the poorer rural communities in the agricultural heartland, lead to their outmigration, and with it destroy the cascade irrigation based civilization of our country.
\end{abstract}

The cause of the disease remains unknown. However, a global pattern has emerged: an insidious form of chronic interstitial nephritis leading to CKD, predominantly affecting poorer, rural, male farmers, in agrochemical intense forms of cultivation, where sun exposure is common, and therefore vulnerable to dehydration [3]. In Sri Lanka, proposed aetiological toxins include cadmium, arsenic, fluoride, aluminium, and most recently glyphosate [4-6]. The likely critical role played by agrochemicals in this context has prompted calls for re-naming Chronic Kidney Disease of Unknown Aetiology (CKDu) as Chronic Agrochemical Nephropathy (CAN), analogous to the term analgesic nephropathy given to recognize the role of anti-inflammatory drugs in causing kidney disease [7].

Though a precise cause has eluded investigators, individual physicians, organizations and research teams (notably from Kandy and Peradeniya) have used available evidence to launch interventions which should be commended. Committees from the level of the cabinet and below, have fast-tracked interventions such as provision of safe water to affected communities, minimizing use of agrochemicals, enhancing social support, and improving health services [8]. The recommendations to the health sector (some of which are being implemented) includes the establishment of population screening and surveillance systems, developing a research agenda, augmenting existing services (e.g. improvement to the Renal Care and Research Center at Anuradhapura), and providing a coherent package of information to the public. It is therefore opportune for the 'curative services' to align with these recommendations and respond effectively and rapidly to the crises. Such a plan will minimize and reduce the immense suffering of these poorer rural communities, at least in the short term.

The points given below are a few proposals relevant especially for the 'curative services' and is intended to facilitate further discussions among professional organizations such as the Sri Lanka Medical Association, the College of Physicians and the Association of Nephrologists of Sri Lanka, the state institutions and relevant Ministries. Though NCP is highlighted in the paper, the disease has probably spread a wider area, and similar interventions will soon be required in other regions of the country.

\section{Services}

Service provision: We do not have precise figures on the number affected by CKD (and CKDu) in the country. This will soon be overcome with the establishment of a surveillance system by the Epidemiology Unit. Recent estimates are that almost 20,000 have died from CKDu [9]. The numbers with the disease are probably much higher, considering the fact that a majority have minimal symptoms until advanced renal failure.

Despite these drawbacks, physicians and nephrologists working in the affected areas have struggled with limited resources to provide a wide range of services $[10,11]$. Providing kidney transplants (KT) for these growing numbers is an impossible task, considering the capacity in the country where less than 5000 transplants have been done since its introduction in 1985. Therefore, while KT remains a long-term strategy, the priority short-term option is to install dialysis services rapidly throughout the affected areas, i.e. chronic haemodialysis (CHD) and continuous ambulatory peritoneal dialysis (CAPD), the relative costs of which are already known [12]. CHD centers could be installed in smaller hospitals, an approach used in Malaysia to tackle the high prevalence CKD from diabetes and hypertension [13,14]. Considering the remoteness of some of the affected villages, we need a combination of these options: those living closer to dialyses centres receive regular CHDs, while those in distant locations get CAPD. Estimates and projections of cases and numbers requiring renal replacement therapies, and potential costs based on patient recruitment, could all be simulated by health economists to develop econometric models that will guide policymakers. 
The network of renal services in the smaller hospitals should be complemented and linked by a dedicated nephrology specific referral system to the main hospitals (e.g. Anuradhapura and Polonnaruwa). The latter should ensure continuous and dedicated nephrology services with adequate number of specialists in adult and paediatric nephrology, and transplantation.

Guidelines for entry to renal replacement therapy at a national level: There appears to be gross inequalities in access to nephrology services in the country. Patients in the NCP have relatively less opportunity to enter a renal replacement therapy programme (of dialysis facilities and transplantation) compared to those in other major cities (e.g. Colombo). In order to overcome this, a coordinated national level renal replacement programme has to be launched that aims at providing similar levels of access to those affected by CKD. It is not acceptable that Sri Lankan farmers are deprived of facilities for renal transplantation, while the country promotes transplantation for foreigners via medical tourism.

Guidelines for case management: Clear consensus guidelines for the management of those affected by CKDu (or CAN) at different levels of care should be freely available. It is opportune for professional associations such as the Sri Lanka Medical Association, the College of Physicians and the Association of Nephrologists of Sri Lanka to develop consensus guidelines for the management of CKDu, while taking into consideration constraints that exist in the high prevalence areas and inequalities in the availability of renal services in the country. These should be reviewed regularly, based on emerging evidence, for example the possible beneficial effects of enalapril in reducing microalbuminuria [15].

Human Resource Development: Human resource development is a prerequisite to develop effective services to tackle the epidemic. One rapid response is to conduct a series of courses (e.g. certificate or diploma courses) on renal care as a collaborative effort, for example between the Ministry of Health and Universities. These should be competency-based and team-based, and include core clinical skills, accessing social support, ethical issues of allocating resource and palliative care. Such models have been successfully used to tackle HIV/AIDS in resource poor settings [16]. This will give practical training to wide range of staff categories with a focus on the management of CKD, and renal replacement therapy. Undergraduate courses too should include a comprehensive curriculum that includes medical, psychosocial and ethical aspects relating to the epidemic. Other novel approaches include utilization of the wide grass-root level network of preventive services or health volunteers to be trained and deployed to supervise the CAPDs in the community.

Research: The government should consider establishing a permanent centre for academic research in the very heartland of the epidemic. This should focus on fundamental and applied research and support and work closely with the Renal Care and Research Centre of the Ministry of Health. The Rajarata University is the obvious choice with close collaborations and links to other well established researchers and research centers in the country. It is unfortunate that the Rajarata University could not lead the research initiatives of the past.

The research center could be a multi-disciplinary centre and similar to the International Centre for Diarrhoeal Disease Research in Bangladesh that has taken research in the area of diarrheoal diseases to new heights. The University should be given cadre provision for such a centre as well to augment its nephrology research capacities and overcome the apparent lack of a dedicated team of scientists and researchers in nephrology.

The areas for clinical research are too vast to enumerate, but should include at least the following (some of which have already been done): impact of CKD on fetal growth, possible relationship to pregnancy induced hypertension, effect on rate of growth of children and their intellectual growth, impact on renal size, clinical trials on efficacy of drugs to reduce progression, trials comparing different modes of renal support, clinical patterns of CKD based on different aetiologies, psychosocial impacts of CKD on families, economic impact of the epidemic, and cultural beliefs in relation to the disease.

Social welfare: The devastation of communities has been highlighted in several reports [17]. The Province should therefore be given a separate budget to provide for social support to the affected families in the form of a financial support. A separate cadre of Health Social Workers, placed in hospitals is essential to coordinate between the chronically ill patients (including CKD) and the network of social support [18]. Transport costs have figured as very high among those affected. Therefore, subsidized transport is essential, and so are the improvements to the transport in these areas.

Other initiatives: CKD will be with us for generations. The numbers will continue to increase with the epidemic of diabetes, already estimated to have afflicted a million [19]. With it there will be an ever growing population with CKD needing dialysate fluid, fluid for CAPD, and tubing for CAPD. Sri Lanka needs industries that will provide for renal replacement therapy and the manufacturing industries should be encouraged to invest in the areas of need. The Ministry of Technology and Scientific Affairs has recently launched a programme to prioritize an agenda for technological developments in the country, and this maybe one opportunity to highlight these needs.

Management and Financing: Implementing a comprehensive programme will be most efficiently accomplished by a dedicated team with a separate budgetary allocation from the treasury ear-marked to tackle CKD in these Provinces. The budget should be allocated to the Ministry of Health, as well as to the provincial administration which controls most of the 
smaller hospitals in the region. That will enable rapid improvements to the network of healthcare institutions in the form of infrastructure, and provide for recurrent expenditures.

CKDu (or CAN) is the beginning of a national catastrophe, perhaps unparalleled in recent history. The actions, non-actions and complacencies of the scientific community and medical profession will be judged by history. The curative services should develop a comprehensive package of interventions to tackle this national calamity. If not, history will judge physicians as an 'orchestra of Neros' who 'played' when Rajarata was poisoned to extinction.

\section{References}

1. Kudalugoda Arachchi J, Lanerolle RD, Kathriarachchi AK et al. Demographic characteristics of end stage renal disease in Sri Lanka. SLMA 112th academic session 1999.

2. Athuraliya TN, Abeysekera DT, Amerasinghe PH, et al. Prevalence of chronic kidney disease in two tertiary care hospitals: high proportion of cases with uncertain aetiology. Ceylon Medical Journal 2009; 54: 23-5.

3. Almaguer M, Herrera R, Orantes CM. Chronic Kidney Disease of Unknown Etiology in Agricultural Communities. MEDICC Review 2014; 16: 9-15.

4. Wanigasuriya K. Update on Uncertain Etiology of Chronic Kidney Disease in Sri Lanka's North-Central Dry Zone. MEDICC Review 2014; 16: 61-5.

5. Noble A, Amerasinghe P, Manthrithilake H, Arasalingam S. Review of Literature on Chronic Kidney Disease of Unknown Etiology (CKDu) in Sri Lanka. IWMI Working Paper 158. International Water Management Institute 2014.

6. Jayasumana C, Gunatilake S, Senanayake P. Glyphosate, Hard Water and Nephrotoxic Metals: Are they the culprits behind the epidemic of Chronic Kidney Disease of Unknown Etiology in Sri Lanka? International Journal of Environment Research and Public Health 2014; 11: 2125-47.

7. Jayasinghe S. Chronic Kidney Disease of Unknown Etiology Should Be Renamed Chronic Agrochemical Nephropathy. MEDICC Review 2014; 16: 72-4.
8. Hettiarachchi K. Tackling kidney disease in NCP: Ministry Secs to go ahead with improved Action Plan. The Sunday Times, 27 April 2014. http://www.sundaytimes.lk/140427/ news/tackling-kidney-disease-in-ncp-93909.html.

9. Alwis K. Chronic kidney disease - when scientists disagree. Colombo, Sri Lanka: National Academy of Sciences of Sri Lanka. http://nas- srilanka.org/?p=599. Posted 10 August 2013 (accessed 14 August 2014).

10. Dassanayake S. Life as a physician in the heart of the epidemic. Abstracts of the 3rd Foundation Sessions of the Ceylon College of Physicians 2013: 25.

11. Dassanayake R. Challenges in management of CKDuNephrologists' perspective. Abstracts of the 3rd Foundation Sessions of the Ceylon College of Physicians 2013: 25-26.

12. Ranasinghe P, Perera YS, Makarim MFM, et al. The costs in provision of haemodialysis in a developing country: A multi-centered study. BMC Nephrology 2011; 12: 42.

13. Lim TO, Lee DG, Zaki M. Dialysis provision in Malaysia. Medical Journal of Malaysia 2000; 55: 188-95.

14. Lim TO, Goh A, Lim YN, et al. How public and private reforms dramatically improved access to dialysis therapy in Malaysia. Health Affairs 2010; 29: 2214-22.

15. Mendis S. Chronic Kidney Disease; Global and Sri Lankan Perspectives. Key note address National Academy of Sciences. http://nas-srilanka.org/wp-content/uploads/2013/ 08/Keynote-address-Shanthi-Mendis.pdf

16. GrayJ, Fana GT, Campbell TB, Hakim JG, et al. Feasibility and sustainability of an interactive team-based learning method for medical education during a severe faculty shortage in Zimbabwe. BMC Medical Education 2014; 14: 63.

17. Liyanage C, Jayathilake R, de Silva A, et al. Socio-economic impact of CKDu: a preliminary analysis. Sri Lanka Medical Association Research Symposium on Chronic Kidney Disease of Unknown Aetiology (CKDu). SLMA 2012: 17.

18. Jayasinghe S. Illness and Social Protection: An agenda for Action in Sri Lanka. Journal of Social Sciences Sri Lanka Journal of Social Sciences 2010/2011; 33/34: 25-9.

19. Katulanda P, Constantine GR, Mahesh JG, et al. Prevalence and projections of diabetes and pre-diabetes in adults in Sri Lanka - Sri Lanka Diabetes, Cardiovascular Study (SLDCS). Diabetes Medicine 2008; 25: 1062-9. 